\title{
Análise epidemiológica comparativa entre sífilis congênita e outras sífilis no estado do Rio de Janeiro nos últimos 10 anos
}

\section{Comparative epidemiological analysis between congenital syphilis and other syphilis in the state of Rio de Janeiro in the last 10 years}

Juliana Lopes Dias ${ }^{\dagger *}$, Felipe Teixeira Freitas ${ }^{\dagger}$, Rafael Spagnol de Almeida ${ }^{\dagger}$, Catharina Ferreira da Costa Magalhães ${ }^{\dagger}$, Eduarda Gomes do Nascimento ${ }^{\dagger}$, Bruno Cezario Costa Reis ${ }^{\ddagger}$

Como citar esse artigo. Dias, J.

L; Freitas F.T; De Almeida, R.S.;

Magalhães, C. F.C.; Nascimento, E.G.;

Reis, B.C.C. Análise epidemiológica comparativa entre sífilis congênita e outras sífilis no estado do Rio de Janeiro nos últimos 10 anos. Revista de Saúde. 2020 Jul./Dez.; 11 (2): 49 - 54

\section{Resumo}

A sífilis é uma infecção sexualmente transmissível que afeta grande parte da população sexualmente ativa, tem diagnóstico e tratamento facéis e apresenta diferentes formas de transmissão e de apresentação. No caso desse estudo, foi separada em sífilis congênita e em outras sífilis a partir da coleta de dados no DATASUS - Sistema de Informações Hospitalares/ Sistema Único de Saúde (SIH/SUS) de 2010 a 2019 e em artigos relevantes publicados entre 2001 e 2020. O objetivo do estudo foi analisar o panorama da sífilis no estado do Rio de Janeiro nos últimos 10 anos, por meio da comparação entre os casos de SC e outras sífilis. Sob essa ótica, visou-se correlacionar a epidemiologia atual com os resultados obtidos e esclarecer o padrão de acometimento da doença utilizando informações importantes, como número de internações, taxa de mortalidade, valor total, sexo, faixa etária, padrão socioeconômico e nível de escolaridade. Foi possível concluir que o número de casos dessa doença têm aumentado em número em ambas apresentações: sífilis adquirida e congênita. Além disso, tem modificado seu padrão de acometimento ao longo dos anos, o que se observa na manifestação tanto em pacientes mais jovens quanto em mais idosos. Assim, é necessário melhorar e ampliar o diagnóstico e o tratamento para diminuir o número de acometidos.

Palavras-chave: Sífilis, Infecção Sexualmente Transmissível, Epidemiologia

\section{Introdução}

A sífilis é uma doença causada pela bactéria Treponema pallidum e está presente no grupo das Infecções Sexualmente Transmissíveis (ISTs) tratáveis e curáveis. Devido a patologia apresentar diferentes manifestações clínicas, divide-se em: primária, secundária, terciária e congênita, sendo esta última de notificação compulsória ${ }^{1}$.

Por ser uma IST, sua transmissão se dá através da relação sexual pelo contato direto com a lesão altamente contagiosa, denominada cancro: lesão única, indolor e localizada principalmente na genitália externa, onde houve o local de entrada da bactéria, caracterizando a sífilis primária².

A secundária se caracteriza principalmente por erupções cutâneas, causadas pela disseminação hematogênica oriunda dos microrganismos do cancro original. Nessa fase, ainda pode estar presente o condiloma plano, que é uma lesão papular modificada e

${ }^{\dagger}$ Discente do Curso de Medicina da Universidade de Vassouras, RJ, Brasil.

* Docente do Curso de Medicina da Universidade de Vassouras, RJ, Brasil.
} 
constitui a lesão mais infectante da sífilis ${ }^{2}$.

$\mathrm{Na}$ hipótese de sífilis terciária, os pacientes que não evoluem para cura, desenvolvem acometimento em outros órgãos como cérebro, coração, ossos e olhos ${ }^{2}$.

Por outro lado, a sífilis congênita (SC) acontece por transmissão vertical, com taxa de transmissão elevada que pode chegar a $100 \%$, mas diminui consideravelmente se o diagnóstico e o tratamento forem feitos corretamente durante a gestação ${ }^{3}$. Nesse caso, pode haver malformações fetais e até aborto. Por esse motivo, o diagnóstico precoce, principalmente nas gestantes, é de extrema importância $\square$.

Diante disso, todas as mulheres grávidas devem realizar os testes para descartar a presença de sífilis, e aquelas com maior risco de contrair a doença devem realizar o exame novamente na $28 \square$ semana de gestação $\square$.

Os testes fornecidos de forma gratuita em toda a rede de atenção primária são o treponêmico (FTA-ABS) e o não treponêmico(VDRL) $\square$. No tangente ao tratamento, utiliza-se a penicilina $G$ benzatina, variando apenas a dose entre uma forma e outra de apresentação ${ }^{1}$. A única diferença na medicação está na neurossífilis, na qual é utilizada a penicilina cristalina, isso porque necessita de altas concentrações no líquor $\square$. É importante, também, que o VDRL seja reavaliado após 6 e 12 meses, no caso de sífilis precoce e, na tardia, em 12 a 24 meses, a fim de avaliar o sucesso da terapia com declínio de pelo menos 4 vezes nos títulos $\square$.

Segundo dados da Organização Mundial da Saúde (OMS), na América do Norte, os casos de sífilis primária e secundária aumentaram de 0,9 para 1,9 por 100000 mulheres entre 2012 e 2016 e, de forma semelhante, os níveis de infecção de sífilis congênita cresceram de 8,4 para 15,5 casos por 100000 nascimentos, totalizando um aumento de $86,9 \%$ nesse mesmo período ${ }^{8}$. Além disso, estudos indicam que a sífilis congênita corresponde a, aproximadamente, $50 \%$ dos recém-nascidos com sequelas físicas, sensoriais e de desenvolvimento e que fatores socioeconômicos tem grande influência sobre sua incidência $\square^{\prime} \square$.

Sendo assim, apesar de ter diagnóstico acessível no sistema público, além de tratamento de baixo custo, os dados apresentados revelam que essa doença parece caminhar no sentido contrário à erradicação.

Portanto, dada a relevância dessa doença, é de suma importância analisar sua epidemiologia, a fim de evidenciar suas novas formas de acometimento, encontrar os entraves para que o seu controle seja alcançado e discutir os fatores que a fazem, ainda hoje, um relevante problema de saúde pública.

Objetivou-se analisar o panorama da sífilis no estado do Rio de Janeiro nos últimos 10 anos, por meio da comparação entre os casos de sífilis congênita e de outras formas de sífilis. Sob essa ótica, visou-se correlacionar a epidemiologia atual com os resultados obtidos e esclarecer o padrão de acometimento da doença, utilizando informações importantes como número de internações, taxa de mortalidade, valor total, sexo, faixa etária, padrão socioeconômico e nível de escolaridade.

\section{Materiais e Métodos}

Foi realizada uma revisão sistemática de literatura no período de 2001 a 2020, utilizando como ferramentas de pesquisa o Google Acadêmico, Scielo e Pubmed, onde foram utilizadas os seguintes descritores: sífilis, sífilis congênita, infecção sexualmente transmissível e epidemiologia.

Os critérios de seleção foram publicações dos últimos 10 anos, incluindo revisões de literatura, artigos originais e boletins epidemiológicos, que abordassem a sífilis adquirida, sífilis congênita e/ou sua epidemiologia. A partir desse levantamento, foram selecionados 34 artigos, dos quais apenas 23 foram inclusos. Foram excluídos aqueles em que não havia acesso na íntegra por meio eletrônico e aqueles em que não havia versão em português ou inglês.

Associada à revisão, foi feita uma coleta observacional, descritiva e transversal de dados disponíveis no DATASUS - Sistema de Informações Hospitalares do Sistema Único de Saúde (SIH/SUS) do Ministério da Saúde, sendo avaliado no período de janeiro de 2010 a dezembro de 2019 as internações, taxa de mortalidade, valor total, sexo e faixa etária.

\section{Resultados}

O estado do Rio de Janeiro, de acordo com os dados do DATASUS, registrou 16.539 internações por SC no período de 2010 a 2019, com taxa de mortalidade de 0,24 e gasto total de R $\$ 7.542 .744,65$. Desse total, o número aumentou consideravelmente com o passar dos anos, registrando $32,56 \%$ dos casos de 2010 a 2014; e $67,43 \%$ de 2015 a 2020 (conforme tabela 1). Esses números demonstram que as internações por SC tiveram um considerável aumento nos últimos cinco anos. Além disso, embora não haja tanta diferença entre os sexos, o estudo apresenta que a maioria afetada foi a população feminina, com $54,67 \%^{1} \square$.

Em contrapartida, os demais casos de sífilis somaram 1.316 no intervalo de tempo analisado, com taxa de mortalidade de 0,91 e gasto total de $\mathrm{R} \$ 494.802,15$. Nesse período, as internações pela doença tiveram números praticamente constantes, não aumentando tanto quanto os casos de SC. Contudo, vale ressaltar que as faixas etárias de acometimento vêm se alterando, visto que a doença está afetando cada vez 
mais os jovens.

Evidencia-se que menores de 20 anos representaram 824 do total, sendo que de 2010 a 2014 o número de afetados com menos de 20 anos foi 44,29\%, enquanto de 2015 a 2019 foi $55,70 \%$, ou seja, o número de internações nos mais jovens também cresceu nos últimos cinco anos. Esse incremento deve-se ao aumento de infectados principalmente menores de 1 ano e de 15 a 19 anos de idade (conforme tabelas 2 e 3$)^{1} \square$.

Outro dado importante, foi o de internações a partir dos 60 anos, que somou 64 do total, sendo 71,87\% destes a partir 2015, essa também é uma mudança no padrão de acometimento da sífilis e reflete, principalmente, os casos da terciária, em que a neurossífilis compõe um importante diagnóstico diferencial de demência em idosos. O sexo mais afetado também foi o feminino com $56,99 \%{ }^{1} \square$.

\section{Discussão}

A SC apresentou, comparativamente, maior número de internações no sistema público de saúde. A justificativa para isso pode estar relacionada aos recémnascidos, tendo em vista que demandam internação para cuidados de sequelas e complicações da doença, quando esta não foi tratada durante a gestação ou quando não houve êxito no tratamento.

O estudo Nascer do Brasil evidencia também a realidade social dessa doença, isso porque, segundo ele, a SC está associada a fatores como: menor escolaridade materna, cor da pele negra ou parda e maior proporção de fatores de risco para prematuridade, bem como ao início mais tardio do pré-natal, menor número de consultas e menor realização de exames sorológicos, demonstrando que mulheres com infecção pela sífilis são mais vulneráveis socialmente ${ }^{3}$. Esses dados são relevantes principalmente porque o tratamento, se realizado corretamente, previne cerca de $97 \%$ dos casos de transmissão vertical ${ }^{11}$.

Portanto, o acesso a cuidados médicos eficazes é essencial para diminuir os número da $\mathrm{SC}$, motivo pelo qual sua taxa de incidência é um bom instrumento de avaliação da atenção básica à saúde, uma vez que reflete diretamente a qualidade da assistência perinatal ${ }^{12},{ }^{13}$. Corroborando esses fatos, os principais estudos mundiais apontam que 70 a $90 \%$ dos casos de SC são consequência do acompanhamento pré-natal inadequado ${ }^{1} \square$.

Paralelo a isso, dados do Ministério da Saúde mostram um aumento de $300 \%$ no número de casos de sífilis em gestantes no Brasil, entre 2010 e 2016 . Dentre as causas estão o aumento do número de testes rápidos realizados e o aumento do número de notificações via Sistema Nacional de Agravos de Notificação (SINAN). Contudo, a subnotificação ainda é grande, revelando
Tabela 1: Internações, valor total e taxa de mortalidade exibida por ano por sífilis congênita., no período de 2010 a 2019, no Estado do Rio de Janeiro.

\begin{tabular}{cccc}
\hline Ano & $\begin{array}{c}\text { Internações } \\
\text { (número absoluto) }\end{array}$ & Valor Total (R\$) & $\begin{array}{c}\text { Taxa de } \\
\text { mortalidade }\end{array}$ \\
\hline 2010 & 663 & $262.628,88$ & 0,15 \\
2011 & 812 & $385.435,69$ & 0,37 \\
2012 & 978 & $493.982,79$ & 0,20 \\
2013 & 1310 & $498.735,49$ & 0,31 \\
2014 & 1623 & $704.161,80$ & 0,25 \\
2015 & 2077 & $982.358,65$ & 0,29 \\
2016 & 2115 & $887.124,11$ & 0,33 \\
2017 & 2348 & $1.059 .144,40$ & 0,04 \\
2018 & 2246 & $1.177 .393,06$ & 0,27 \\
2019 & 2367 & $1.091 .779,78$ & 0,21 \\
\hline TOTAL & $\mathbf{1 6 5 3 9}$ & $7.542 .744,65$ & $\mathbf{0 , 2 4}$ \\
\hline
\end{tabular}

Lista de Morb CID-10: Sífilis Congênita Fonte: SIH/SUS ${ }^{1} \square$

Tabela 2: Internações, segundo faixa etária, por outras formas de sífilis no Estado do Rio de Janeiro, no período de 2010 a 2014.

\begin{tabular}{cc}
\hline Faixa Etária & Internações \\
\hline Menores de 1 ano & 209 \\
1 a 4 anos & 2 \\
5 a 9 anos & 2 \\
10 a 14 anos & 4 \\
15 a 19 anos & 67 \\
20 a 29 anos & 116 \\
30 a 39 anos & 51 \\
40 a 49 anos & 26 \\
50 a 59 anos & 30 \\
60 a 69 anos & 9 \\
70 a 79 anos & 7 \\
80 anos ou mais & 2 \\
\hline Total & 606 \\
\hline
\end{tabular}

Lista de Morb CID-10: Outras sífilis Fonte: SIH/SUS ${ }^{1} \square$ 
Tabela 3: Internações, segundo faixa etária, por outras formas de sífilis no Estado do Rio de Janeiro, no período de 2015 a 2019.

\begin{tabular}{cc}
\hline Faixa Etária & Internações \\
\hline Menores de 1 ano & 416 \\
1 a 4 anos & 13 \\
5 a 9 anos & 6 \\
10 a 14 anos & 4 \\
15 a 19 anos & 20 \\
20 a 29 anos & 53 \\
30 a 39 anos & 47 \\
40 a 49 anos & 57 \\
50 a 59 anos & 48 \\
60 a 69 anos & 28 \\
70 a 79 anos & 13 \\
80 anos ou mais & 5 \\
\hline TOTAL & 710 \\
\hline
\end{tabular}

Lista de Morb CID-10: Sífilis Congênita Fonte: SIH/SUS ${ }^{1} \square$

que a sífilis durante a gestação é ainda mais grave do que indicam os dados, o que contribui para aumentar também a $\mathrm{SC}^{1} \square$.

Esse panorama faz com que haja maior incidência de prematuridade, restrição de crescimento intrauterino, abortamento e até óbito perinatal ${ }^{12}$. Além disso, o diagnóstico precoce é prejudicado, visto que dois terços dos neonatos são assintomáticos, isso porque os sintomas são tardios, podendo levar meses ou anos para se tornarem evidentes ${ }^{12}$. As principais consequências são sequelas como surdez, cegueira e retardo mental, que podem ser evitadas com tratamento, mas são irreversíveis ${ }^{1} \square$.

Por outro lado, no caso da sífilis adquirida, foi constatado que houve aumento no número de adolescente e até de crianças, e há ainda o fato de que as outras apresentações da sífilis nem sempre são tratadas e são subnotificadas, sendo evidente que seu número é ainda maior do que os dados que o DATASUS informam.

Enquanto os dados do DATASUS demonstram que no estado do Rio de Janeiro há maior prevalência da sífilis adquirida em menores de 20 anos, dados do Ministério da Saúde revelam que houve, no Brasil, um aumento da taxa de incidência para todas as faixas etárias, ressaltando a tendência mais acentuada de aumento entre 20 e 29 anos, faixa que, em 2018, obteve
163,3 casos por 100000 habitantes ${ }^{1} \square$. Ressalta-se que, nesse caso, também estão relacionados menor nível de escolaridade e maior número entre as pessoas de pele negra e parda, embora, devido ao não preenchimento correto do prontuário por muitos profissionais, haja um déficit na colheta dessas informações ${ }^{1} \square$.

Nesse sentido, tanto no estado do Rio de Janeiro, quanto no Brasil como um todo, os números dessa doença estão crescendo, podendo-se identificar que a população mais acometida é de jovens.

Os registros de casos de sífilis em maiores de 60 anos representam um dado alarmante, principalmente devido ao acometimento de determinados órgãos já citados, pela sífilis terciária. Dentre esses, a neurossífilis se manifesta, após 10 a 20 anos da infecção inicial não tratada, como uma meningoencefalite progressiva ${ }^{1} \square$. Nesse caso, a clínica é heterogênea, a depender da evolução e do estágio em que a doença se encontra, mas, geralmente, tem início insidioso e, se não tratada, piora progressivamente até se tornar semelhante a um estado demencial orgânico qualquer ${ }^{1} \square$.

Considerando que em $70 \%$ dos pacientes em que há invasão das meninges a infecção desaparece mesmo sem tratamento, o aumento do número de idosos com neurossífilis na última década reflete problemas no sistema de saúde, tanto no que diz respeito ao diagnóstico quanto ao tratamento $1 \square$. Por esse motivo, a neurossífilis, hoje, se encontra como um dos principais diagnósticos diferenciais em idosos com quadro demencial.

Outro ponto importante a ser discutido sobre a sífilis é a necessidade do acompanhamento adequado após o uso da medicação, tendo em vista que há a possibilidade de reativação e também de reinfecção. Nesses casos, se os exames forem repetidos o retratamento pode ser realizado precocemente, evitando a evolução, as complicações e também que o paciente continue transmitindo, o que impacta diretamente nos números da doença.

No caso da reativação, ocorre por tratamento inadequado, quando não há adesão do doente, mas também é um alerta para neurossífilis e, nesse caso, a punção lombar de líquor deve ser realizada e a medicação reavaliada ${ }^{2} \square$. Nesse sentido, é importante acompanhar principalmente os paciente coinfectados pelo HIV, isso porque mesmo após um tratamento adequado eles apresentam maior risco de falha sorológica ${ }^{21,22}$.

Além disso, também existe a cicatriz sorológica (serofast), que acomete 15 a $41 \%$ dos infectados. Nessa situação, o VDRL continua reagente após a conclusão da terapêutica, mas com queda prévia da titulação em pelo menos duas diluições ${ }^{23,2} \square$. Ou seja, apesar de não tornarse não reativo, não representa uma falha terapêutica ${ }^{2} \square$. Esse quadro se encaixa apenas se uma nova exposição for descartada e, portanto, o retratamento não é necessário, 
principalmente porque estudos sugerem que apenas $27 \%$ desses pacientes atingem a cura sorológica ${ }^{2} \square, 23$.

Considerando o panorama exposto no presente trabalho, o gasto com a sífilis, bem como o número de infectados, representam um dado preocupante para a saúde pública no Brasil.

\section{Considerações Finais}

A sífilis, mesmo sendo uma IST considerada passível de cura, ainda representa um problema de saúde pública, considerando-se que obteve um maior acometimento na população com o passar dos anos, além de ter mudado seus padrões, o que não era esperado para uma doença com testes diagnósticos disponíveis no SUS e tratamento de baixo custo.

Em paralelo ao aumento no número global de afetados, a sífilis também passou por modificações no padrão de faixa etária, dado que pacientes mais jovens, incluindo adolescentes, crianças e menores de 20 anos, estão sendo cada vez mais acometidos, assim como há mais idosos que não foram tratados corretamente, ou nem mesmo tratados desde a infecção inicial, manifestando os sintomas da sífilis terciária. Concomitante a isso, estão as internações por SC, que representaram, no presente estudo, $92,62 \%$ do total, demonstrando também aumento considerável com o passar dos anos. Evidencia-se, portanto, a insuficiência na testagem durante a gestação ou até mesmo a ausência do cuidado para evitar a transmissão para o feto.

Embora as taxas de mortalidade por essa doença não sejam altas, os gastos da saúde pública dedicados ao seu tratamento poderiam ser minimizados através de diagnóstico e tratamento precoces.

Ademais, é possível concluir que as populações socialmente vulneráveis, como aquelas de baixa escolaridade, negras e pardas apresentam maior incidência da doença.

Analisando todos esses dados, o principal ponto do estudo é evidenciar que, infelizmente, não nos encaminhamos hoje para um melhor controle da doença, além de que a epidemiologia vem se modificando e afetando cada vez mais pessoas ao longo da década analisada.

Visto isso, é necessário, para melhorar o panorama da doença, que as gestantes e mulheres com suspeita de sífilis, assim como as que forem grupo de risco, sejam testadas nas unidades básicas de saúde, para melhorar o acesso dos mais vulneráveis e diminuir as disparidades sociais que foram observadas.

Além disso, aquelas com positividade serão tratadas e deverão tratar os parceiros, mesmo que os agentes de saúde tenham que contribuir nesse caso com a busca ativa.

Visando minimizar a afecção de jovens, a educação sexual se mostra de suma importância, especialmente no que diz respeito ao uso correto de preservativos, devendo ser um tema ensinado desde a escola até a vida acadêmica, o que evitaria essa e outras ISTs.

Por fim, sabe-se que os pacientes devem fazer o acompanhamento dos níveis de VDRL após o tratamento, porém, há aqueles que não o fazem após a conclusão ou nem mesmo concluem o tratamento. Sendo assim, é extremamente importante que os exames sejam refeitos em todos os pacientes, mesmo que isso necessite, novamente, de apoio através da busca ativa pelos agentes de saúde. Isso é importante porque o retratamento poderá ser feito quando necessário e, nos casos da cicatriz sorológica, os baixos níveis de VDRL não serão confundidos com uma nova infecção, poupando recursos da saúde pública.

\section{Referências}

1 - Souza MJ, Mendes AB, Souza MM, Nogueira AF, Carvalho PC, Lopes VR. Perfil Epidemiológico Da Sífilis Gestacional: Clínica e Evolução de 2012 a 2017. Sanare - Revista de Políticas Públicas [Internet]. 2018 [Acesso em: 2020 Mar 19];17(2). Disponível em: https://sanare.emnuvens.com.br/ sanare/article/view/1257.

2 - Facco A, Dias F, Pontes G, Righetto L, Oliveira L, Bolzan P, Weber SR, Costenaro RGS. Sífilis: Um Saber Necessário Para Quem Luta Pela Vida, Seres Que Cuidam E Que São Cuidados [Internet]. 2002 [Acesso em: 19 March 2020]. Disponível em: https://periodicos.ufn.edu.br/index.php/ disciplinarumS/article/view/851/795.

3 - Domingues R, Leal M. Incidência De Sífilis Congênita E Fatores Associados À Transmissão Vertical Da Sífilis: Dados Do Estudo Nascer No Brasil. Cadernos de Saúde Pública [Internet]. 2016 [Acesso em 2020 Mar 19]; 32(6). Disponível em: https://doi.org/10.1590/0102-311X00082415.

4 - Harmon ED, Robertson EW. Syphilis: A Growing Concern. The Nurse Practitioner [Internet]. 2020 [Acesso em: 2020 Mar 19];. Disponível em: https://doi.org/10.1097/01.NPR.0000558159.61349.cb

5 - Ghanem KG, Ram S, Rice PA. The Modern Epidemic of Syphilis. The New England Journal of Medicine [Internet]. 2020 [Acesso em: 2020 Mar 20];. Disponível em: https://doi.org/10.1056/NEJMra1901593.

6 - Silva DAR, Alves IGFG, Barros MPT, Dorneles FV. Prevalência De Sifilis Em Mulheres [Internet]. 2017 [Acesso em: 2020 Mar 21];. Disponível em: http://revista.cofen.gov.br/index.php/enfermagem/article/view/891/401.

7 - Organização Mundial de Saúde. WHO Guidelines For The Treatment Of Treponema Pallidum (Syphilis). 2016 p. 1-60. [Acesso em: 2020 Mar 19] Disponível em: https://apps.who.int/iris/bitstream/handle/10665/249572/97 89241549806-eng.pdf;jsessionid=727C0B0A1EA32B50457187D56C478C $9 \mathrm{~A}$ ? sequence $=1$.

8 - Rowe CR, Newberry DM, Jnah AJ. Congenital Syphilis: A Discussion of Epidemiology, Diagnosis, Management, and Nurses' Role in Early Identification and Treatment. Adv Neonatal Care. 2018 [Acesso em: 2020 Mar 19] ;18(6):438 $\square$ 445. Disponível em: https://doi.org/10.1097/ ANC.0000000000000534.

9 - Beck E, Souza M. 2018. Práticas De Enfermagem Acerca Do Controle Da Sífilis Congênita / Nursing Practices On The Control Of Congenital Syphilis. Revista Online de Pesquisa, [online] pp.19-24. [Acesso em: 2020 Mar 21]. Disponível em: http://http://www.seer.unirio.br/index.php/ cuidadofundamental/article/viewFile/7596/6581.

10 - DATASUS (SIH-SUS)- avaliado de jan de 2010 a dez 2019, avaliando internações, ano processamento, óbitos, valor total, faixa etária, sexo. Acesso 
em: $21 / 03 / 2020$.

11 - Blencowe H, Cousens S, Kamb M, Berman S, Lawn J. Lives Saved Tool Supplement Detection And Treatment Of Syphilis In Pregnancy To Reduce Syphilis Related Stillbirths And Neonatal Mortality. BMC Public Health11, S9 [Internet]. (2011) [Acesso em 2020 Maio 11];. Disponível em: https://doi. org/10.1186/1471-2458-11-S3-S9.

12 - De Lorenzi DRS, Madi JM. Sífilis Congênita Como Indicador de Assistência Pré-natal. Rev. Bras. Ginecol. Obstet. [Internet]. 2001 Dec [Acesso em: 2020 Mai 19]; 23(10):647-652. Disponível em: http://dx.doi. org/10.1590/S0100-72032001001000006.

13 - Saraceni V, Espinosa MA. Relação Entre a Cobertura da Estratégia Saúde da Família e o Diagnóstico de Sífilis na Gestação e Sífilis Congênita [Internet]. 2011 [Acesso em: 2020 Mai 19]. Disponível em: https://www. scielosp.org/article/csp/2012.v28n3/490-496/.

14 - Vilela LSCDAL, Souza GS, Vasconcelos BM, Gama CR, Silva LSM, Cerqueira TMG, Santos RFEP, Norberto DS. O pré-natal como ferramenta na prevenção da sífilis congênita: uma revisão integrativa da literatura. Brazilian Journal of Health Review [Internet]. 2020 [Acesso em: 19 Mar 2020];:16161623. Disponível em: http://file:///Users/phelps/Downloads/1414-4518-1PB.pdf.

15 - Ministério da Saúde (Brasil), Secretaria de Políticas de Saúde. Boletim epidemiológico: Sífilis 2017. Brasília (DF); 2017. Acesso em: 19/03/2020.

16 - Albuquerque G, Chaves E, Sampaio L, Dias K, Patrocínio M, Vasconcelos S. Complicações Da Sífilis Congênita: Uma Revisão De Literatura [Internet]. Pesquisa.bvsalud.org. 2014 [Acesso em: 2020 Maio 11]. Disponível em: https://pesquisa.bvsalud.org/portal/resource/pt/lil-737060.

17 - Ministério da Saúde (Brasil), Secretária de Políticas de Saúde. Boletim Epidemiológico: Sífilis 2019. Brasília (DF); 2019. Acesso em: 19/03/2020

18 - Reimer HRC, Ferreira CL. Prevalência e Estudo Neuropsicológico De Transtornos Cognitivos e Demências Decorrentes De Neuroinfecções Em Hospital De Referência [ebook]. 2010 [Acesso em: 2020 Mar 25]. Disponível em: https://repositorio.bc.ufg.br/rede/handle/tde/1842.

19 - Regazzi A. C. J., Bottino G. Sífilis: Diagnóstico, Tratamento e Controle. An. Bras. Dermatol. [Internet]. 2006 Mar [Acesso em: 2020 Maio 11] ; 81( 2 ): 111-126. Disponível em: https://doi.org/10.1590/S036505962006000200002 .

20 - Ministério da Saúde (Brasil), Secretaria de Vigilância em Saúde. Protocolo Clínico e Diretrizes Terapêuticas para Atenção Integral às Pessoas com Infecções Sexualmente Transmissíveis (IST). Brasília (DF); 2018. Acesso em: 19/03/2020.

21 - Ghanem KG, Erbelding EJ, Wiener ZS, Rompalo AM. Resposta Sorológica Ao Tratamento Da Sífilis Em Pacientes HIV Positivos E Negativos Para HIV Atendidos Em Clínicas De Doenças Sexualmente Transmissíveis. Sex Transm Infect. 2007 [Acesso em: 2020 Mar 19]; 83 2): 97-101. Disponível em: https://doi.org/10.1136/sti.2006.021402.

22 - González-López JJ, Guerrero MLF, Luján R, Tostado SF, Górgolas M, Raquena L. Fatores que Determinam a Resposta Sorológica ao Tratamento em Pacientes Com Sífilis. Clin Infect Dis. 2009 [Acesso em: 2020 Mar 19];49(10):1505-1511. Disponível em: https://doi.org/10.1086/644618.

23 - Clement ME, Okeke NL, Hicks CB, Treatment of Syphilis A Systematic Review [Internet]. 2014 [Acesso em: 2020 Mar 19]. Disponível em: https:// doi.org/10.1001/jama.2014.13259. 